\title{
Validity of Transesophageal echocardiography in estimating left ventricular filling pressures in anaesthetised patients undergoing cardiac surgery
}

\author{
Sudheer Vanga ${ }^{1}$, Aveek Jayant ${ }^{1}$, Rakhi Balachandran ${ }^{1}$, and praveen varma ${ }^{1}$ \\ ${ }^{1}$ Amrita Institute of Medical Sciences and Research Centre
}

March 18, 2021

\begin{abstract}
Background :The aim of this study was to validate whether pulmonary capillary wedge pressure estimated using transoesophageal echocardiography in anaesthetised patients receiving mechanical ventilation can be comparable to gold standard method of estimating PCWP by using pulmonary artery catheter. Methods : A cross sectional validity study conducted in a tertiary care cardiac centre in south India over a period of 6 months from February 2016 - August 2016 among 100 patients undergoing cardiac surgery where intraoperative TEE and pulmonary artery catheter used. In anesthetised patients, after the insertion of TEE probe, the following echocardiography ratios were calculated: 1) SF = S / S + D (systolic fraction of pulmonary venous flow 2) Systolic / diastolic (S / D ratio) 3) E / Ea 4) E / Vp 5) E / A ratio , for three consecutive cardiac cycles and averaged by two independent and blinded observers. All the echo measurements were taken pre cardiopulmonary bypass at end expiration and during a stable period of hemodynamics. Simultaneously PCWP was recorded from PAC. The ability of these variables to predict PCWP was tested for previously determined cutoffs as per extant guidelines and validity parameters such as sensitivity, specificity, predictive value of positives, predictive value of negatives \& accuracy were computed. Conclusion : Use of $\mathrm{E} / \mathrm{A}, \mathrm{DT}, \mathrm{S} / \mathrm{D}, \mathrm{SF}$ ratio \& $\mathrm{E} / \mathrm{Vp}$ reveal poor predictive capacity for detecting high left sided filling pressures. The use of $\mathrm{E} / \mathrm{Ea}$ is reasonable to predict an elevated PCWP especially if the cohort predominantly comprises of patients with normal ejection fraction
\end{abstract}

\section{Introduction :}

The pulmonary artery catheter (PAC) has historically been used to measure the pulmonary capillary wedge pressure (PCWP $)^{1}$. This measurement is considered as a surrogate of left atrial pressure (LAP) and, by extension, left ventricular end-diastolic pressure. Various studies have shown that PCWP and LAP correlate well in patients with cardiovascular disease ${ }^{2,3}$. Estimation of LAP is a core component of the evaluation of left ventricular diastolic function ${ }^{4}$. Elevated left ventricular filling pressures predict long-term adverse outcomes, even in patients with normal ejection fractions ${ }^{5}$. A restrictive diastolic filling pattern predicts major morbidity and mortality in patients undergoing cardiac surgery ${ }^{6}$.

The use of the PAC has been questioned in the perioperative period with some studies unable to clearly demonstrate clinical benefit. This has resulted in declining use in the perioperative period ${ }^{7}$. Alternative methods like echocardiography has been used for the noninvasive estimation of PCWP and LAP. Transthoracic echocardiography (TTE) is non-invasive and is therefore of considerable appeal as a measurement tool. Various Doppler measurements have been made in healthy patients and in different disease states, and their correlation and agreement with PCWP is reasonable. Transesophageal echocardiography (TEE) is used routinely in cardiac surgery for the evaluation of hemodynamic conditions, left ventricular function and valvular function. Few studies 8,9 were attempted to validate correlation and agreement of Doppler measurements using TEE and PCWP in anaesthetised patients receiving mechanical ventilation. It should not be assumed 
that TTE validation will automatically apply to patients using TEE, although successful validation would be of great clinical benefit and reduce reliance on the pulmonary artery catheter.

\section{MATERIALS AND METHODS:}

\section{Study design:}

This is a cross sectional validity study conducted in a tertiary care cardiac centre over a period of 7 months in 2016 after getting approval by institutional review board and a total of 100 patients were enrolled in the study. The need for patient consent was waived off as the study was purely an observational study. Based on the sensitivity of cut off points for different $n$ variables (variables SF, E/A, DT, S/D) reported in an earlier publication ${ }^{10}$, minimum sample size is estimated as follows : $\mathrm{SF}<0.6$ with $10 \%$ allowable error and $95 \%$ confidence, minimum sample size is 73 , for E/A $>2$ with $10 \%$ allowable error \& $99 \%$ confidence $\mathrm{n}$ is 50 , for $\mathrm{S} / \mathrm{D}<1.5$ with $15 \%$ allowable error \& $99 \%$ confidence $\mathrm{n}$ is 65 . Based on the above results, 100 cases were included 100 cases in the study.

\section{Selection of study participants :}

Inclusion criteria: Consecutive patients undergoing cardiac surgery, where intra operative TEE and the PAC were used.

Exclusion criteria : Patients were not studied if they were in atrial fibrillation, had aortic or mitral valve lesions classified as being greater than mild or if transesophageal echocardiography was contraindicated

\section{CONDUCT OF STUDY:}

All patients received their previously prescribed cardiac medications before surgery in accordance with institutional practice. Anesthesia management was standard and typically consisted of intravenous propofol at induction, isoflurane in air-oxygen for maintenance and fentanyl for analgesia. Standard monitoring included electrocardiography, invasive arterial and central venous pressure, pulmonary artery catheter monitoring, pulse oximetry, capnography, temperature, urine output and transesophageal echocardiography was used in all patients.

The balloon was inflated for repeated measures of PCWP. Measurements were made at end-expiration. The zero pressure reference point was at the level of the mid-axillary line. After insertion of transesophageal echo probe, echocardiography measurements for three consecutive cardiac cycles were analysed and averaged by two independent and blinded observers. All the echo measurements were taken pre cardiopulmonary bypass at end expiration and during a stable period of hemodynamics. Simultaneously PCWP was recorded from PAC. A pulmonary capillary wedge pressure [?] $15 \mathrm{mmHg}$ was used to define elevated left atrial pressure ${ }^{10}$.

Previously published formulae derived from TTE:

Equation 1: $\mathrm{PCWP}=31.7-0.134 \mathrm{X} \mathrm{DT}^{11}$

Equation 2: $\mathrm{PCWP}=8.8 \mathrm{X} \mathrm{E} / \mathrm{Vp}-1.9^{12}$

Equation 3: $\mathrm{PCWP}=18.4+(17.1 \mathrm{X} \mathrm{E} / \mathrm{A})^{13}$

Equation 4: $\mathrm{PCWP}=0.97 \mathrm{X} \mathrm{E} / \mathrm{Ea}+4.34^{14}$

Equation 5: $\mathrm{PCWP}=3.91 \mathrm{E} / \mathrm{Vp}+4.92^{14}$

Equation 6: PCWP $=35-0.39 \mathrm{SF}$ (where \% systolic fraction

is calculated using velocity time integrals rather than

peak velocities $)^{15}$.

The following measurements were taken from transesophageal echocardiography: 
1. Left ventricular inflow Doppler spectral display was obtained from the mid-oesophageal four-chamber view with the sample volume placed at the mitral valve tips. Measurements included peak early diastolic velocity, E ; late velocity, $\mathrm{A}(\mathrm{cm} / \mathrm{sec})$; deceleration time of E-wave, DT and A wave duration $(\mathrm{msec})$.

2. Pulmonary venous spectral Doppler was recorded with the sample volume placed $0.5-1 \mathrm{~cm}$ into the left pulmonary vein from the mid-esophageal four-chamber view. Measurements included peak velocities of the systolic $\mathrm{S}$ and diastolic D, atrial reversal AR velocities $(\mathrm{cm} / \mathrm{sec})$ and $\mathrm{AR}$ duration $(\mathrm{m} \mathrm{sec})$.

3. Left ventricular flow propagation velocity, $\mathrm{Vp}(\mathrm{cm} / \mathrm{sec})$ was measured from the mid-esophageal fourchamber view as the first aliasing velocity during early mitral filling. Measurements were taken from the mitral valve leaflets to $4 \mathrm{~cm}$ distally into the left ventricle.

4. Tissue Doppler imaging was obtained from the mid-esophageal four-chamber view, with the cursor placed over the basal lateral wall at mitral annulus level. Measurements included early left ventricular filling, $\mathrm{Ea}(\mathrm{cm} / \mathrm{sec})$.

The following echocardiography ratios were calculated:

1. $\mathrm{SF}=\mathrm{S} / \mathrm{S}+\mathrm{D}$ (systolic fraction of pulmonary venous flow) 2. E / Vp 3. E / Ea 4. Systolic / diastolic $(\mathrm{S} / \mathrm{D})$ ratio 5. E / A ratio

\section{Demographic details :}

A total of 100 patients who are undergoing cardiac surgery were recruited for the study.

The patient characteristics are as follows: Among n,100 patients, $11 \%$ are females and $89 \%$ are males. The majority of patients (66\%) had normal left ventricular ejection fraction ([?]50\%). Nearly all the study participants $(96 \%)$ underwent sole coronary artery bypass grafting (CABG), all of these procedures were performed off cardiopulmonary bypass. Some patients also underwent adjunct procedures under CPB and a solitary patient was operated upon for aortic dissection. Most of the patients had pulmonary capillary wedge pressures less than $15 \mathrm{~mm} \mathrm{Hg}$. PCWP [?] $15 \mathrm{~mm} \mathrm{Hg}$ is considered as abnormal.

\section{Statistical details :}

The ability of ordinal variables $(\mathrm{E} / \mathrm{A}, \mathrm{S} / \mathrm{D}, \mathrm{E} / \mathrm{Vp})$ to predict PCWP was tested for previously determined cutoffs as per extant guidelines. Based on diastolic function guidelines ${ }^{4}$, sensitivity and specificity were calculated for prefixed cutoff values i.e., $(\mathrm{E} / \mathrm{A}<1, \mathrm{E} / \mathrm{A} 1-2, \mathrm{E} / \mathrm{A}>2, \mathrm{~S} / \mathrm{D}<1 \& \mathrm{E} / \mathrm{Vp}>2.5)$. For continuous data, optimal cut off values were determined by constructing ROC curves i.e., for echocardiographic variables ( SF, E/Ea, DT).

As per new revision of diastolic function guidelines $2016^{16}$, evaluation of diastolic function should proceed on different lines in patients with normal versus depressed left ventricular systolic function because of high prevalance of diastolic dysfunction in the latter. Accordingly, when the pretest probability of diastolic dysfunction is much higher in patients with systolic dysfunction, any attempt to distinguish presence or absence of diastolic dysfunction should stress on specificity.

For patients with normal systolic function, cut off values using ROC curve analysis were calculated by focusing on sensitivity. So, for variables (SF, E/Ea, DT), cut off values were calculated based on both maximizing sensitivity and specificity. In all the cases, validity parameters such as sensitivity, specificity, predictive value of positives, predictive value of negatives \& accuracy were computed.

\section{Specific parameters:}

E/A: The overall correlation between E/A and PCWP (graph 1) was weak with a Pearson correlation coefficient of $0.27(\mathrm{p}=0.005)$. As per guidelines, $\mathrm{E} / \mathrm{A}<1$ is associated with normal LAP. In our study series, 2 patients had PCWP [?]15 even though E/A $<1$, this indicates a sensitivity of $16.7 \%$ and specificity of $61.4 \%$ in detecting elevated PCWP. E/A $>2$ is associated with elevated LAP. In our study ,4 patients had 
PCWP $<15$ even though E/A $>2$ which indicates a sensitivity of $33.3 \%$ and specificity of $95.5 \%$ in identifying elevated PCWP.

E/A,1-2 is indeterminate in detecting elevated PCWP. In our study E/A,1-2 had a sensitivity of $50 \%$ and specificity of $43.2 \%$ in detecting elevated PCWP.

DT: The correlation between DT and PCWP (Graph 2) was demonstrated to be weak with a Pearson correlation coefficient of $-0.064(\mathrm{p}=0.526)$. The area under the ROC curve for using DT to predict elevated PCWP ([?]15mmHg) is 0.47 (C.I: 0.31-0.63). Maximising the specificity, the optimum threshold value of DT to predict elevated PCWP ([?]15 mm Hg) is 196 with a sensitivity of $8.3 \%$ and specificity of $80.7 \%$ with accuracy of $72 \%$. Maximising the sensitivity, the optimum threshold value of DT to predict elevated PCWP ([?]15 mm Hg) is 116.5 with a sensitivity of $83.3 \%$ and specificity of $23.9 \%$ with accuracy of $31 \%$.

S/D: The correlation between S/D and PCWP was weak with a pearson correlation coefficient of -0.086 (p $=0.393$ ). As per guidelines, $\mathrm{S} / \mathrm{D}<1$ is associated with elevated LAP. In our study, 21 patients had $\mathrm{PCWP}<15$ even though S/D $<1$ showing a sensitivity of $58.3 \%$ and specificity of $76.1 \%$ in detecting elevated PCWP.

SF RATIO: The correlation between SF ratio and PCWP (Graph 4) is weak with a pearson correlation coefficient of -0.143 ( $\mathrm{p}=0.393$ ). The area under the ROC curve for using SF ratio to predict elevated PCWP ([?]15mmHg) is 0.35 (C.I: $0.14-0.57$ ). Maximising the specificity, the optimum threshold value of SF ratio to predict elevated PCWP ([?]15 mm Hg) is 0.6 with a sensitivity of $25 \%$ and specificity of $81.8 \%$ with accuracy of $75 \%$.Maximising the sensitivity, the optimum threshold value of SF ratio to predict elevated PCWP ([?]15 $\mathrm{mm} \mathrm{Hg}$ ) is 0.39 with a sensitivity of $83.3 \%$ and specificity of $2.3 \%$ with accuracy of $12 \%$.

E/Ea: The correlation between E/Ea ratio and PCWP (Graph 5)is weak with a pearson correlation coefficient of 0.181 ( $\mathrm{p}=0.071)$. The area under the ROC curve for using $\mathrm{E} / \mathrm{Ea}$ to predict elevated PCWP $([?] 15 \mathrm{mmHg})$ is 0.79 (C.I: 0.67- 0.92).

Maximising the specificity, the optimum threshold value of E/Ea to predict elevated PCWP ([?]15 mm $\mathrm{Hg}$ )

(Graph 4) is 11.55 with a sensitivity of $50 \%$ and specificity of $84.1 \%$ with accuracy of 80 .

Maximising the sensitivity, the optimum threshold value of E/Ea to predict elevated $\mathrm{PCWP}([?] 15 \mathrm{~mm} \mathrm{Hg})$ is 9.2 with a sensitivity of $91.7 \%$ and specificity of $63.6 \%$ with accuracy of $67 \%$.

Guidelines suggest that an E/Ea $>14$ is associated with elevated PCWP. In our series, an E/Ea $>14$ had a sensitivity of $33.3 \%$ and specificity of $93.2 \%$ with accuracy of 86 to detect an elevated PCWP [?]15 mm Hg.

$\mathbf{E} / \mathbf{V p}$ : The correlation between E/Vp ratio and PCWP (Graph 6) is weak with a pearson correlation coefficient of 0.221 ( $\mathrm{p}=0.027)$. Guidelines suggest that an $\mathrm{E} / \mathrm{Vp}>2.5$ is associated with elevated PCWP. In our study series, 9 patients had $\mathrm{PCWP}<15$ with $\mathrm{E} / \mathrm{Vp}>2.5$, showing a sensitivity of $33.3 \%$ and specificity of $89.8 \%$ to detect an elevated PCWP [?]15 $\mathrm{mm} \mathrm{Hg}$.

\section{Discussion :}

Pulmonary artery catheterization was shown to be harmful and increase the resource utilization in ICU ${ }^{17}$. However it can be argued that, this may be true of mixed intensive care unit populations. The study may not be applicable to the patients with cardiac disease, in particular cardiac surgical population. As recently as 2011, Schwann et al ${ }^{18}$, demonstrated a similar outcome specific to the cardiac surgical population. These authors showed that PAC use increased mortality and organ specific complications. At the same time there has been a parallel increase in the use of transesophageal echocardiography in the perioperative cardiac surgical population. The current guidelines ${ }^{19}$, recommends TEE use in all open heart procedures, a distinct difference from the previous version of the guidelines which limited use to specific populations. While TEE can offer excellent hemodynamic monitoring it may not be able to measure vascular pressures as accurately as invasive catheterization. Given the continuous endeavor to limit the use of invasive vascular devices on account of multiple risks, it is reasonable to develop echocardiography based surrogates of estimating static pressures such as the pulmonary artery systolic pressure, the left ventricular end diastolic or left atrial 
pressure. Our investigation is yet another attempt in this direction but specifically targeted at the surgical population with a focus on the intra- operative period.

A hundred subjects, predominantly undergoing myocardial revascularization without cardiopulmonary bypass constituted the study population. A majority of the population $(66 \%)$ had normal left ventricular systolic function. Also, when pulmonary artery wedge pressures were categorized to be 'high' ([?]15 mm $\mathrm{Hg}$ ), only $12 \%$ of patients had numerical values above this limit.

The Doppler evaluation of transmitral flow has been traditionally used to categorise patients with abnormalities of diastolic function, and, by extension higher left sided filling pressure ${ }^{4}$. According to 2009 ASE diastolic function guidelines, transmitral flow Doppler was used to classify patients into 3 distinct classes $-\mathrm{E} / \mathrm{A}<1, \mathrm{E} / \mathrm{A} 1-2, \mathrm{E} / \mathrm{A}>2$ representing either normal, equivocal or elevated left atrial pressure respectively. This was particularly pertinent to patients with depressed LV ejection fraction. In our study $36 \%$ of population were having $\mathrm{E} / \mathrm{A}<1,56 \%$ have $\mathrm{E} / \mathrm{A} 1-2$ and $8 \%$ were having $\mathrm{E} / \mathrm{A}>2$ suggesting that in this population majority were having equivocal $\mathrm{E} / \mathrm{A}$ values.

In the group with E/A 1-2 , this measure alone could detect only $50 \%$ of patients with elevated PCWP. Specificity was also suboptimal (43.2\%). However a very small number of patients $(6 / 56)$ in the study cohort were having simultaneous values of $\mathrm{E} / \mathrm{A} 1-2$ and $\mathrm{PCWP}>15$. When the $\mathrm{E} / \mathrm{A}>2$, this value was highly specific (95\%) in detecting elevated PCWP but continued to be poorly sensitive(33\%). When the $\mathrm{E} / \mathrm{A}<1$, both sensitivity and specificity were markedly poor. Thus, in accordance with the extant guidelines, transmitral Doppler is extremely low utility in determining PCWP and only can be used in conjunction with other measures such as early mitral annular velocities (Ea), deceleration time or absolute velocity of $\mathrm{E}>50$ $\mathrm{cm} / \mathrm{sec}$.

Another measure used to assess the left ventricular filling status is the ratio of systolic phase to diastolic phase velocities in the pulmonary vein. With decrease in left atrial compliance the proportion changes such that the S/D ratio is less than unity. In the population under study, S/D ratio scored better than the transmitral flow velocity ratio in so far as the sensitivity was higher at $58.6 \%$; however the specificity was lower at $76 \%$.

The majority of patients in the population had normal $\mathrm{EF}$ and the measurement has been de-emphasised both in the current ${ }^{16}$ and the previous EAE/ASE guidelines ${ }^{4}$ for such patients. An extension of this measurement is the systolic faction (SF). In our study the correlation coefficient for systolic fraction and PCWP was weak. In patients with normal $\mathrm{EF}$ a threshold value of SF set at 0.39 was considerably sensitive but was virtually useless in determining truly positive patients. When specificity criteria were used SF was considerably specific in determining elevated filling pressures but was poorly sensitive. It could therefore be of use only in patients with depressed ejection fraction and therefore a much higher pre- test probability of suffering high filling pressures in the left heart. The pattern demonstrated in the study for these three variables (E/A, $\mathrm{S} / \mathrm{D}$ and the $\mathrm{SF}$ ) is entirely consonant with previous descriptions in the literature ${ }^{20}$.

However the cutoff for maximising specificity as determined in our study was higher at 0.6 rather than 0.4 as mentioned in previous standard guidelines ${ }^{4}$, on the subject. It is unknown whether this is on account of the significant change in loading conditions that might ensue from administration of anaesthetics and positive pressure ventilation. Also the PCWP value set in our study at $15 \mathrm{~mm} \mathrm{Hg}$ is not the universal cutoff value to determine elevated filling pressure and is sometimes set at $12 \mathrm{~mm} \mathrm{Hg}$ or higher. The inability to obtain reasonable sensitivity and specificity simultaneously continued for the other measures such as deceleration time and the ratio $\left(\mathrm{E} / \mathrm{V}_{\mathrm{p}}\right)$ of early phase of transmitral flow $(\mathrm{E})$ to mitral propagation velocity $\left(\mathrm{V}_{\mathrm{p}},\right)_{\text {. }}$. Similar considerations such as those that apply to E/A, S/D ratios and the SF apply to these parameters as well. In determining true positives DT with a threshold of $196 \mathrm{msec}$ was able to differentiate patients with a PCWP[?]15 from those with lower value of this variable (specificity $80.7 \%$ but with a sensitivity of $8 \%$ and accuracy of $72 \%$ ). This is replicated elsewhere in the literature ${ }^{21,22}$.

There has been significant focus on measurement of early mitral annular velocities measured from either the lateral or the septal mitral annulus or both. This parameter denoted as Ea is central to determination of the 
presence or absence of diastolic function in patients with normal ejection fraction in the current guideline. Ea velocities have been significantly correlated to the time constant of left ventricular relaxation (tau). They are widely considered to be less loading condition dependent than their blood pool counterpart (E).

The ratio of the two velocities $(\mathrm{E} / \mathrm{Ea})$ with a threshold of 14 has been set as a fairly consistent discriminator of raised vs normal filling pressure in the recently issued guidelines ${ }^{16}$ on the subject, across patients with normal and reduced EF. The important caveat remains that clinical practice guidelines are in themselves not validated and are not therefore extrapolatable.

In our dataset of patients with predominantly preserved $\mathrm{EF}$, the E/Ea was, by far, the most consistent discriminator of raised vs normal filling pressure with areas under the receiver operator characteristic curve of $0.79,95 \%$ confidence interval of (0.67-0.92). The thresholds to diagnose an elevated PCWP with emphasis on specificity was 11.55 which is lower than most of the literature described cutoff values. Even with a sensitivity exceeding $91 \%$ a cutoff set at 9.2 was determined to have a specificity of $64 \%$ with an accuracy of $67 \%$. It is important to note that both thresholds lie in the conventional interval considered to be indeterminate ${ }^{23}$.

There are at least three studies with a focus on the cardiac surgical population on semi invasive estimation of PCWP using echo based assessment; one of the studies only used the E/Ea ratio and was unable to demonstrate adequate correlation between the echo and the invasive pressure surrogate ${ }^{24}$. Yet another study which was not restricted to the intra- operative period alone but also studied preoperative transthoracic echocardiogram derived estimates found that while the pre-operative results were largely consonant with the traditional discourse on the subject the institution of general anaesthesia and the subsequently obtained transesophageal values were grossly discordant. E/Ea lost considerable discriminative power.

Further, the TTE values also performed poorly suggesting that this had less to do with the processes involved in extrapolation from TTE to TEE and vice versa ${ }^{10}$. In the post cardiopulmonary bypass period the $\mathrm{E}$ velocity and the $\mathrm{S}$ velocity also increased steeply for yet unknown reasons. With continued focus on the perioperative use of the E/Ea our study showed better predictive value for the E/Ea than previously reported in the perioperative literature, area under receiver operating characteristic curve (AUROC) of 0.79 vs. 0.68 as previously reported, Cowie $\mathrm{B}$ et.al ${ }^{25}$.

Thus, our study in consonance with the global literature replicates the poor predictive value of transmitral and pulmonary vein Doppler in assessment of high filling pressures. It suggests that the best possible estimate for elevated filling pressure may be made from an assessment of the E/Ea; this is higher than previously reported and should be tested in a larger, prospective population with separate group analyses for patients with normal and reduced ejection fractions respectively.

\section{LIMITATIONS OF THE STUDY:}

The use of a single point estimate, lack of comparison between pre and post coronary artery bypass, the paucity of patients with both reduced ejection fraction and overall high filling pressures clearly limits the generalisibility of these conclusions.

\section{CONCLUSION:}

Use of transmitral flow velocity, deceleration times and pulmonary vein Doppler velocities reveal poor predictive capacity for detecting high left sided filling pressures (PCWP [?]15 $\mathrm{mm} \mathrm{Hg}$ ). The use of E/Ea is reasonable to predict an elevated PCWP especially if the cohort predominantly comprises of patients with normal ejection fraction and where in an overwhelming majority are undergoing myocardial revascularisation without cardiopulmonary bypass. Also, the study, with the caveat that limited patient numbers have been enrolled suggest different cutoff points for this estimate than previously reported.

\section{References:}

1. Cowie, B. S. Does the pulmonary artery catheter still have a role in the perioperative period? Anaesth. Intensive Care $\mathbf{3 9}, 345-55$ (2011). 
2. Nagy, A. I. et al. The pulmonary capillary wedge pressure accurately reflects both normal and elevated left atrial pressure.Am. Heart J. 167, 876-83 (2014).

3. Walston, A. \& Kendall, M. E. Comparison of pulmonary wedge and left atrial pressure in man. Am. Heart J. 86 , 159-64 (1973).

4. Nagueh, S. F. et al. Recommendations for the evaluation of left ventricular diastolic function by echocardiography. J. Am. Soc. Echocardiogr. 22, 107-33 (2009).

5. Nagueh, S. F. Prognostic power of mitral annulus indices of left ventricular diastolic function. J. Am. Heart Assoc. 3, e001012 (2014).

6. Afilalo, J. et al. Incremental value of the preoperative echocardiogram to predict mortality and major morbidity in coronary artery bypass surgery. Circulation 127, 356-64 (2013).

7. Shah, M. R. et al. Impact of the pulmonary artery catheter in critically ill patients: meta-analysis of randomized clinical trials. JAMA 294, 1664-70 (2005).

8. Girard, F. et al. Estimation of the pulmonary capillary wedge pressure from transesophageal pulsed Doppler echocardiography of pulmonary venous flow: influence of the respiratory cycle during mechanical ventilation. J. Cardiothorac. Vasc. Anesth.12, 16-21 (1998).

9. Habu, H. et al. What is the most appropriate variable for estimation of mean pulmonary capillary wedge pressure by transesophageal pulsed Doppler echocardiography? Int. J. Card. Imaging11 , 35-45 (1995).

10. Ali, M. M., Royse, A. G., Connelly, K. \& Royse, C. F. The accuracy of transoesophageal echocardiography in estimating pulmonary capillary wedge pressure in anaesthetised patients. Anaesthesia67 , 122-31 (2012).

11. Martins, S., Soares, R. M., Branco, L., Salomão, S. \& Antunes, A. M. Non-invasive monitoring of pulmonary capillary wedge pressure in heart failure. Eur. J. Heart Fail. 3 , 41-6 (2001).

12. Ueno, Y. et al. Noninvasive estimation of pulmonary capillary wedge pressure by color M-mode Doppler echocardiography in patients with acute myocardial infarction. Echocardiography 19 , 95-102 (2002).

13. Vanoverschelde, J. L. et al. Noninvasive estimation of pulmonary arterial wedge pressure with Doppler transmitral flow velocity pattern in patients with known heart disease. Am. J. Cardiol.75 , 383-9 (1995).

14. Bouhemad, B. et al. Echocardiographic Doppler assessment of pulmonary capillary wedge pressure in surgical patients with postoperative circulatory shock and acute lung injury.Anesthesiology 98 , 1091-100 (2003).

15. Kuecherer, H. F. et al. Estimation of mean left atrial pressure from transesophageal pulsed Doppler echocardiography of pulmonary venous flow. Circulation 82, 1127-39 (1990).

16. Nagueh, S. F. et al. Recommendations for the Evaluation of Left Ventricular Diastolic Function by Echocardiography: An Update from the American Society of Echocardiography and the European Association of Cardiovascular Imaging. J. Am. Soc. Echocardiogr. 29 , 277-314 (2016).

17. Connors, A. F. et al. The effectiveness of right heart catheterization in the initial care of critically ill patients. SUPPORT Investigators. JAMA 276 , 889-97 (1996).

18. Schwann, N. M. et al. Lack of effectiveness of the pulmonary artery catheter in cardiac surgery. Anesth. Analg. 113, 994-1002 (2011).

19. Hahn, R. T. et al. Guidelines for Performing a Comprehensive Transesophageal Echocardiographic Examination: Recommendations from the American Society of Echocardiography and the Society of Cardiovascular Anesthesiologists. J. Am. Soc. Echocardiogr. 26 , 921-964 (2013).

20. Yamamoto, K. et al. Determination of left ventricular filling pressure by Doppler echocardiography in patients with coronary artery disease: critical role of left ventricular systolic function. J. Am. Coll. Cardiol. 
30 , 1819-26 (1997).

21. Haji, D. L. et al. Interatrial Septum Motion but Not Doppler Assessment Predicts Elevated Pulmonary Capillary Wedge Pressure in Patients Undergoing Cardiac Surgery. Anesthesiology 121 , 719-729 (2014).

22. Nishimura, R. A., Abel, M. D., Hatle, L. K. \& Tajik, A. J. Relation of pulmonary vein to mitral flow velocities by transesophageal Doppler echocardiography. Effect of different loading conditions. Circulation 81 , 1488-97 (1990).

23. Otto, catherine $\mathrm{m}$. text book of clinical echocardiography. infifth edition (ed. catherine $\mathrm{m}$ otto) (Elsevier Inc., 2013).

24. Kumar, K. et al. The role of tissue Doppler imaging in predicting left ventricular filling pressures in patients undergoing cardiac surgery: an intraoperative study. Echocardiography30 , 271-8 (2013).

25. Cowie, B., Kluger, R., Rex, S. \& Missant, C. Noninvasive estimation of left atrial pressure with transesophageal echocardiography.Ann. Card. Anaesth. 18, 312-6 (2015).

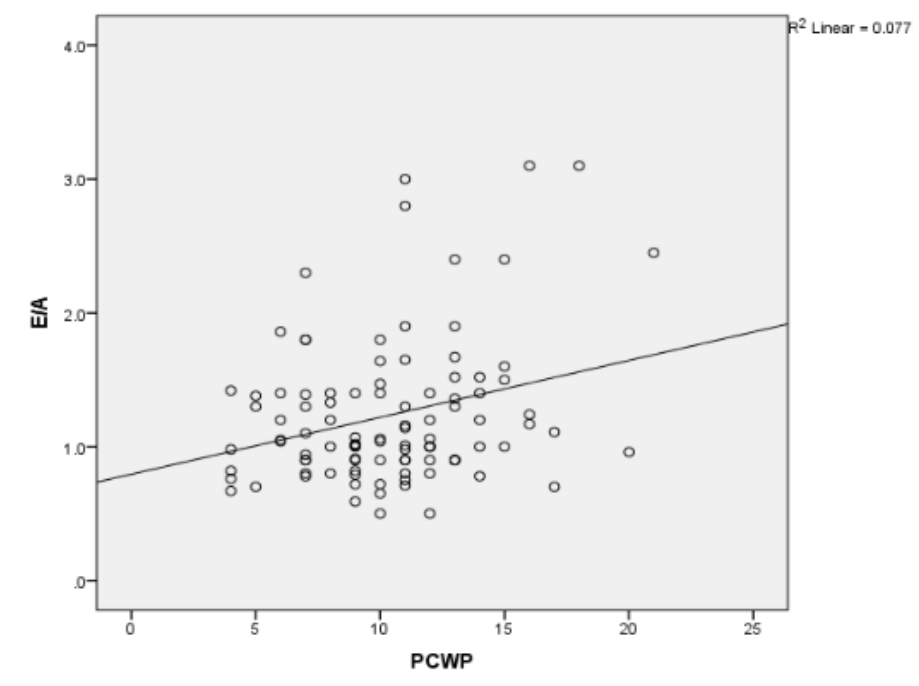

\section{Graph no.1 showing the correlation between E/A and PCWP}




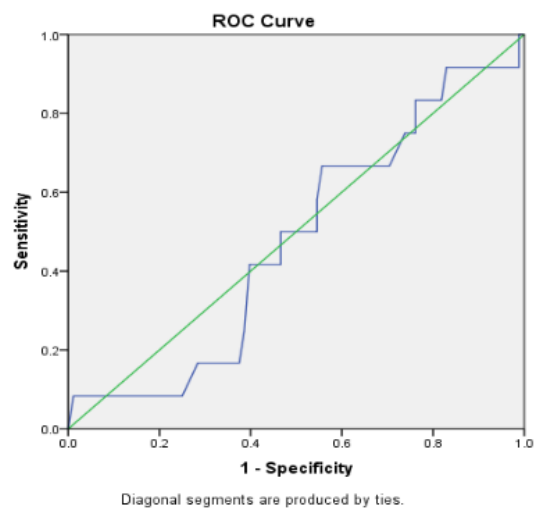

Graph no.2: ROC curve analysis for DT to predict elevated PCWP

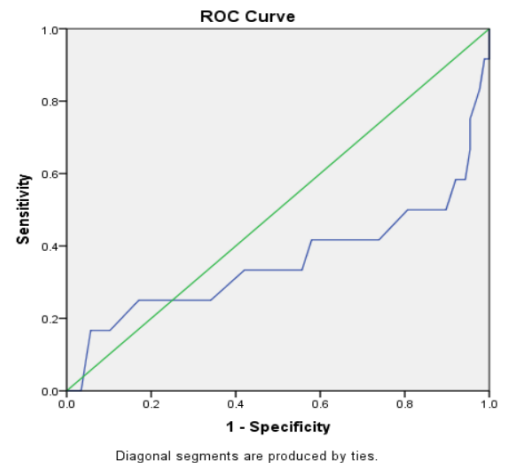

Graph no.3: ROC curve analysis for SF ratio to predict elevated PCWP

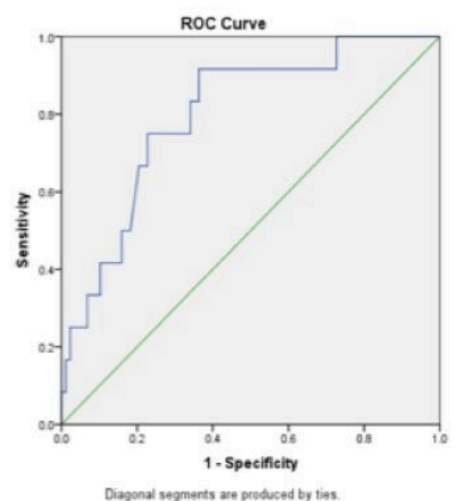

Graph 4:ROC curve analysis for E/Ea to predict elevated PCWP 


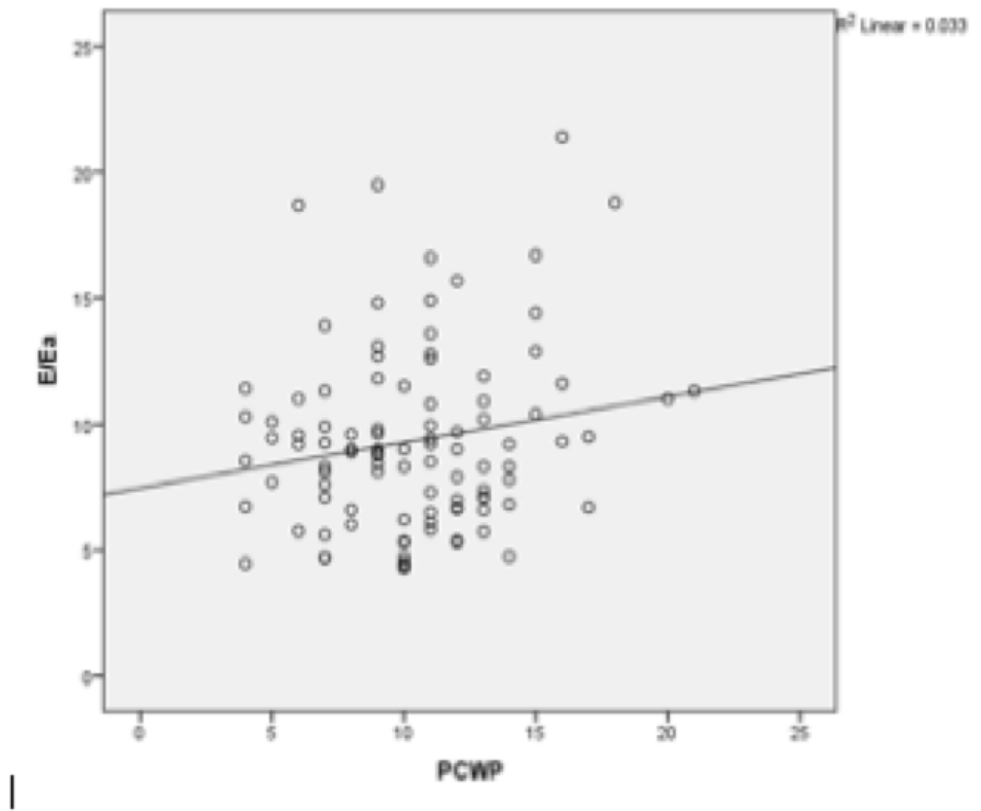

Graph 5: Correlation between E/Ea and PCWP

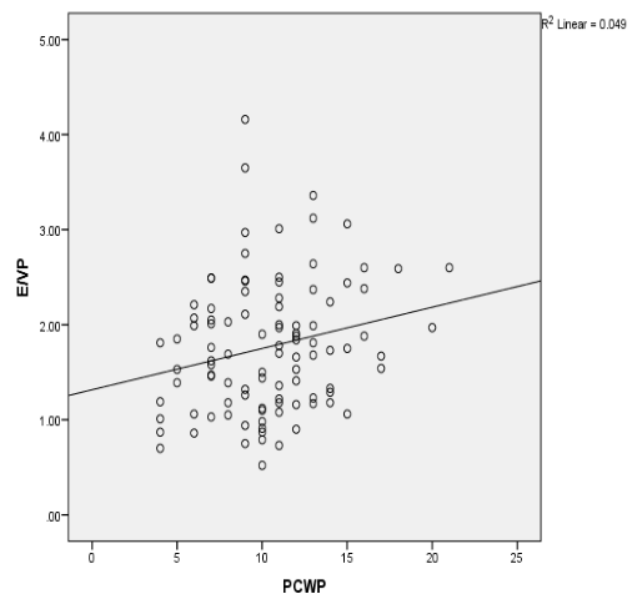

Graph 6: Showing the correlation between E/Vp and PCWP 


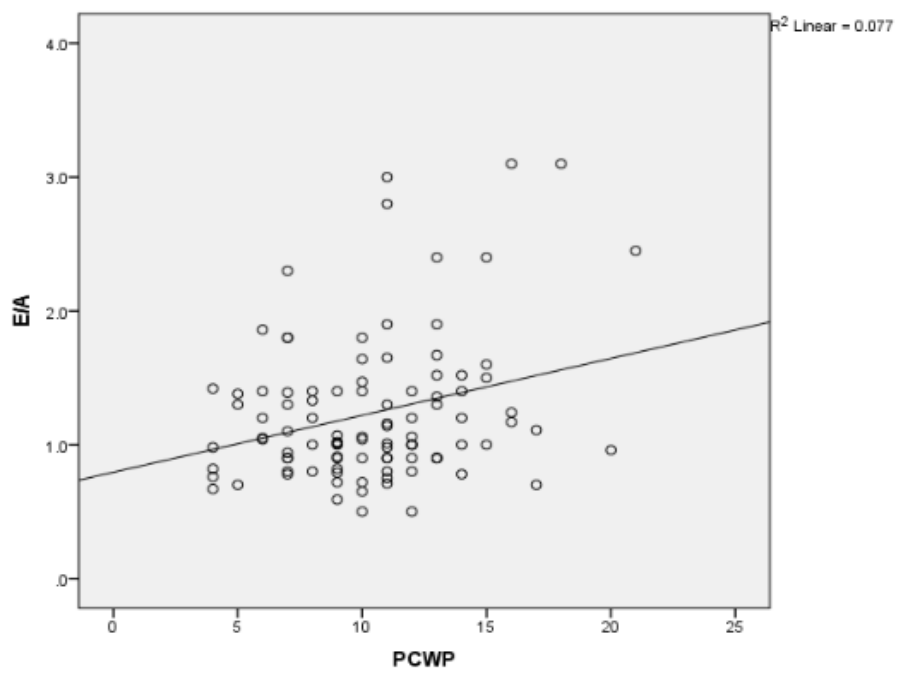

Graph no.1 showing the correlation between E/A and PCWP

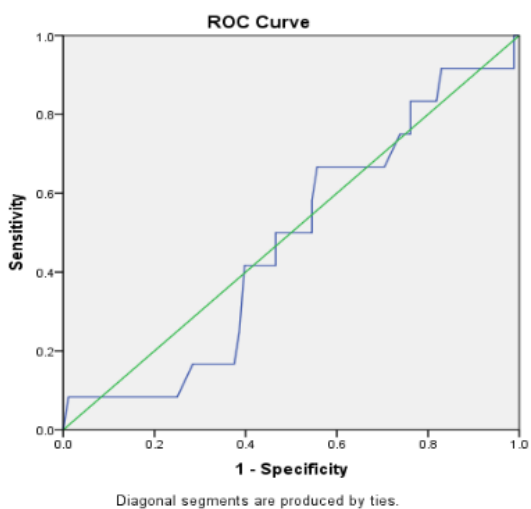

Graph no.2: ROC curve analysis for DT to predict elevated PCWP 


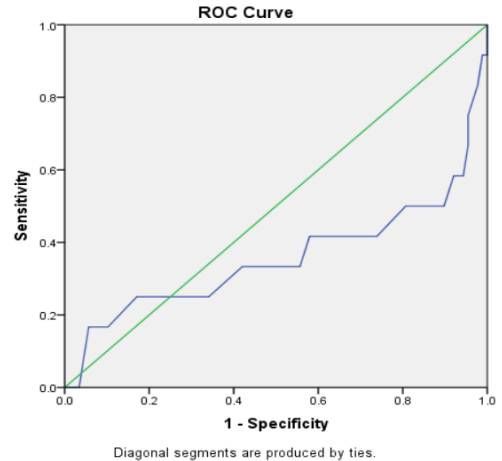

\section{Graph no.3: ROC curve analysis for SF ratio to predict elevated PCWP}

\section{Hosted file}

graph_4-3.pdf available at https://authorea.com/users/402301/articles/514154-validity-oftransesophageal-echocardiography-in-estimating-left-ventricular-filling-pressures-inanaesthetised-patients-undergoing-cardiac-surgery

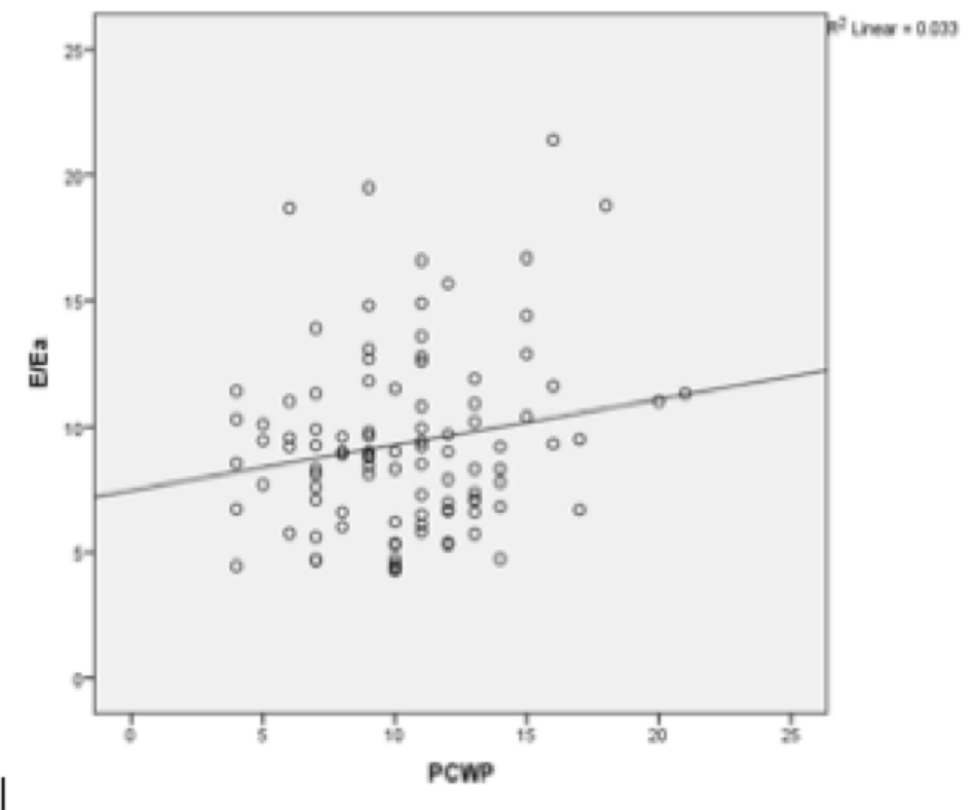

Graph 5: Correlation between E/Ea and PCWP 


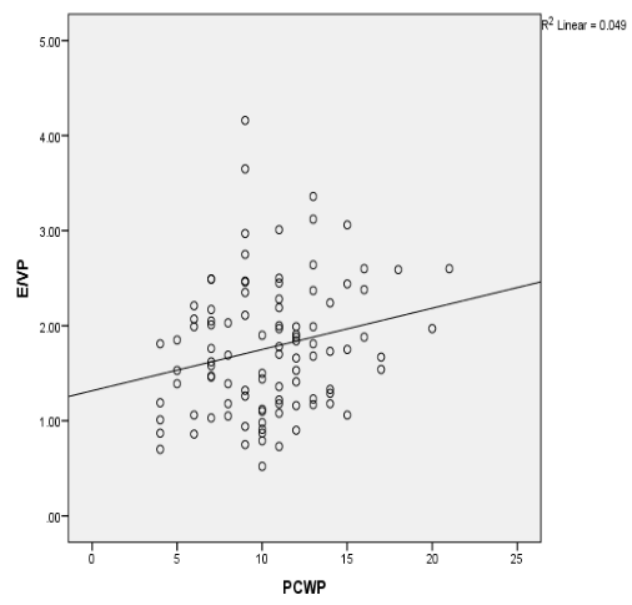

Graph 6: Showing the correlation between E/Vp and PCWP

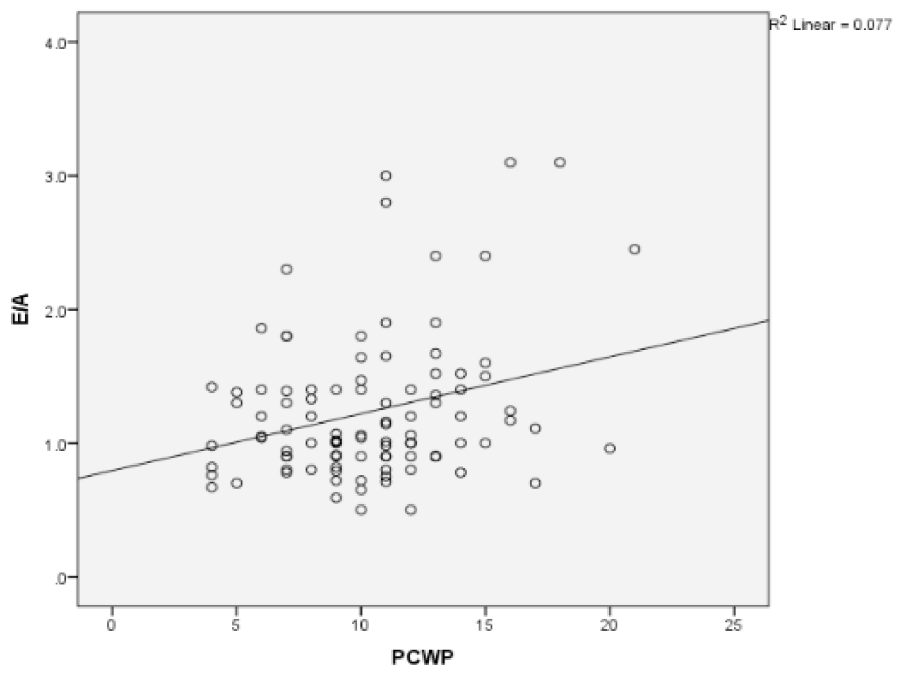

Graph no.1 showing the correlation between E/A and PCWP 


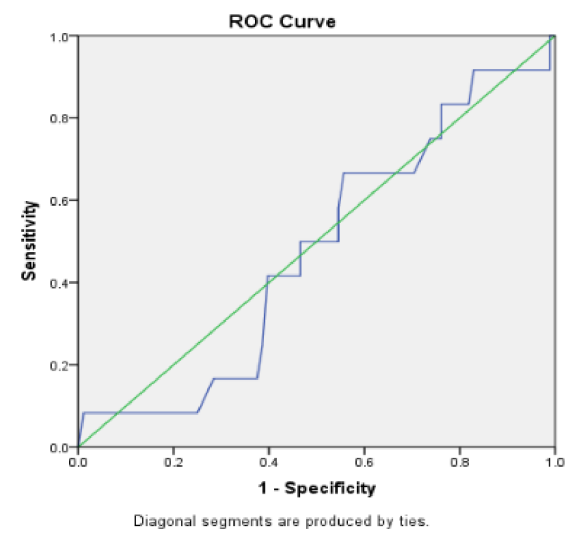

Graph no.2: ROC curve analysis for DT to predict elevated PCWP

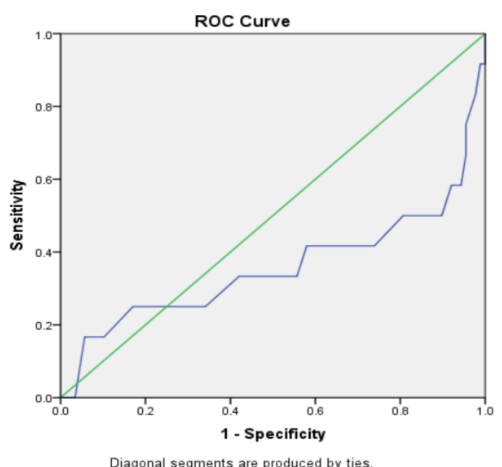

Graph no.3: ROC curve analysis for SF ratio to predict elevated PCWP

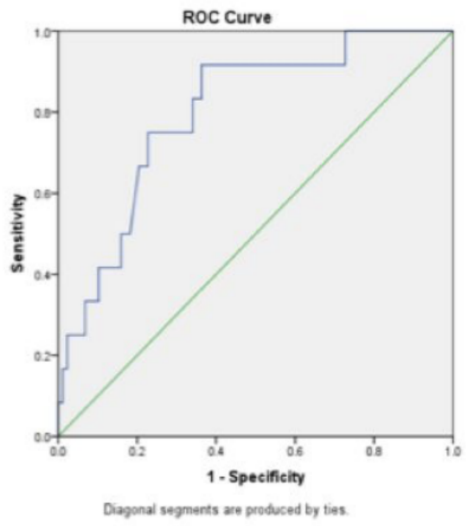

Graph 4:ROC curve analysis for E/Ea to predict elevated PCWP 


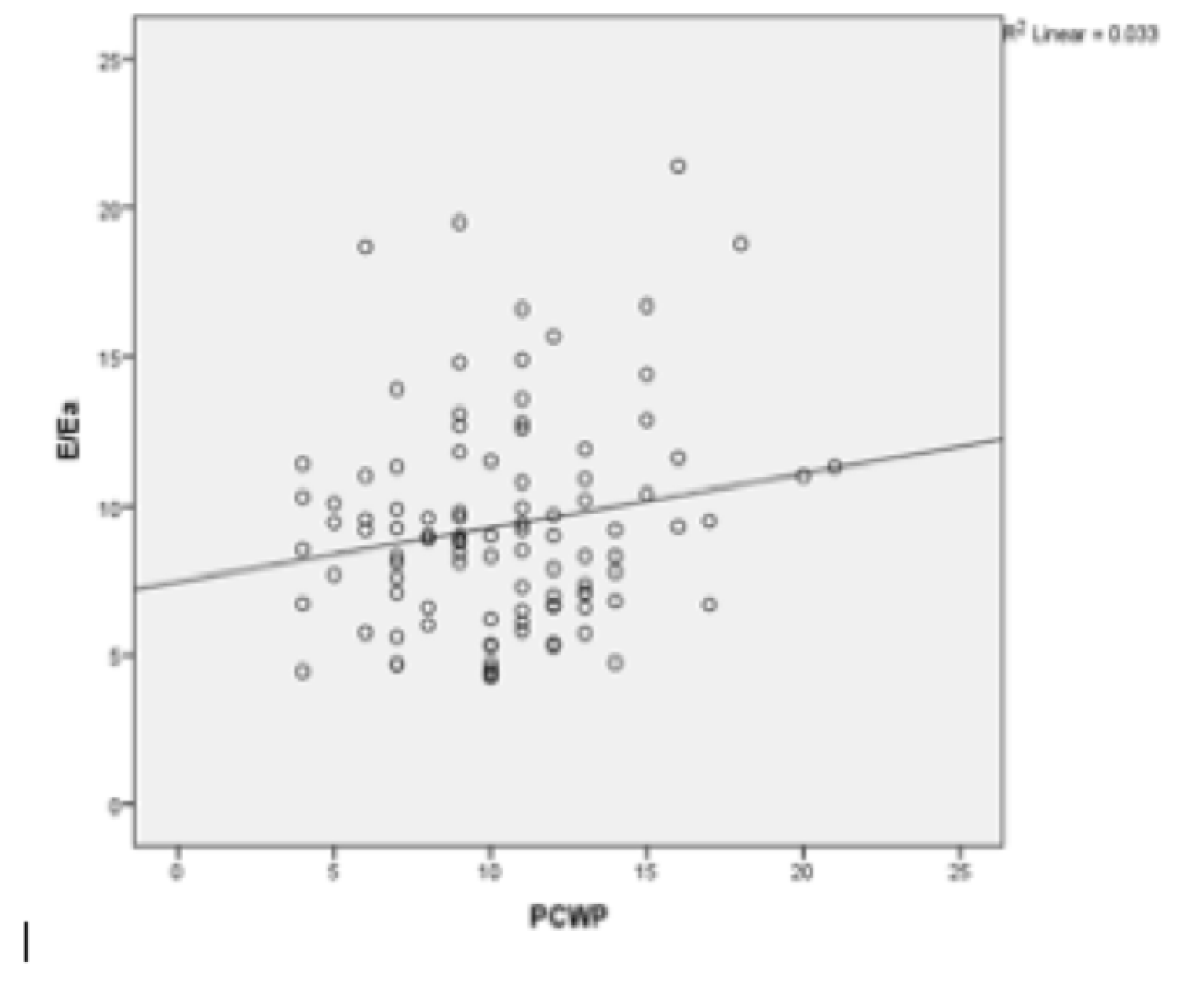

Graph 5: Correlation between E/Ea and PCWP

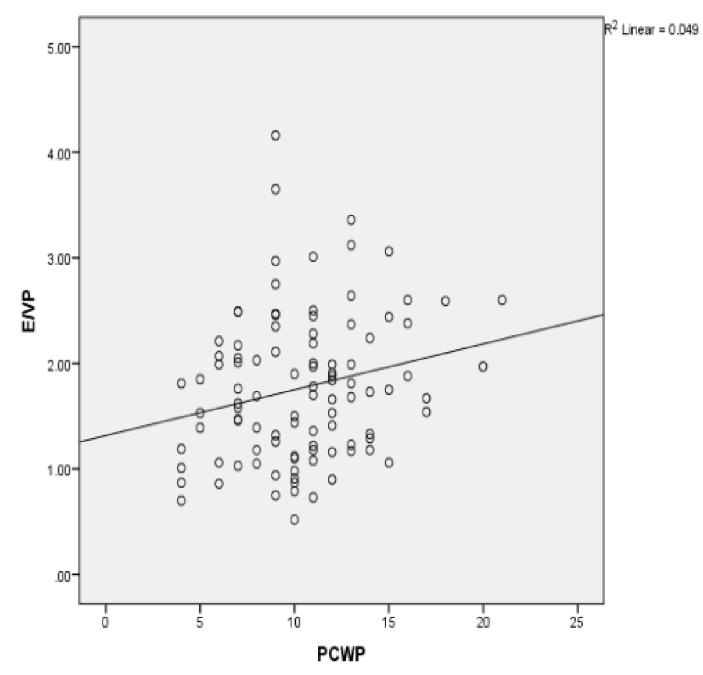

Graph 6: Showing the correlation between E/Vp and PCWP 